\title{
Glucose-dependent insulinotropic polypeptide (GIP) receptor deletion leads to reduced bone strength and quality
}

\author{
Aleksandra Mieczkowska ${ }^{a}$, Nigel Irwin ${ }^{b}$, Peter R. Flatt ${ }^{\mathrm{b}}$, Daniel Chappard ${ }^{\mathrm{a}}$, Guillaume Mabilleau ${ }^{\mathrm{a}, *}$ \\ a LUNAM Université, GEROM-LHEA, Institut de Biologie en Santé, Angers, France \\ b School of Biomedical Sciences, Ulster University, Coleraine, United Kingdom
}

\section{A R T I C L E I N F O}

\section{Article history:}

Received 15 April 2013

Revised 1 July 2013

Accepted 2 July 2013

Available online 10 July 2013

Edited by: David Bur

\section{Keywords:}

GIP

GIPR

Bone quality

Nanoindentation

Bone strength

Gut hormone

\begin{abstract}
A B S T R A C T
Bone is permanently remodeled by a complex network of local, hormonal and neuronal factors that affect osteoclast and osteoblast biology. In this context, a role for gastro-intestinal hormones has been proposed based on evidence that bone resorption dramatically falls after a meal. Glucose-dependent insulinotropic polypeptide (GIP) is one of the candidate hormones as its receptor, glucose-dependent insulinotropic polypeptide receptor (GIPR), is expressed in bone. In the present study we investigated bone strength and quality by three-point bending, quantitative x-ray microradiography, microCT, qBEI and FTIR in a GIPR knockout (GIPR KO) mouse model and compared with control wild-type (WT) animals. Animals with a deletion of the GIPR presented with a significant reduction in ultimate load (--11\%), stiffness $(-16 \%)$, total absorbed $(-28 \%)$ and post-yield energies $(-27 \%)$ as compared with WT animals. Furthermore, despite no change in bone outer diameter, the bone marrow diameter was significantly increased and as a result cortical thickness was significantly decreased by $20 \%$ in GIPR deficient animals. Bone resorption at the endosteal surface was significantly increased whilst bone formation was unchanged in GIPR deficient animals. Deficient animals also presented with a pronounced reduction in the degree of mineralization of bone matrix. Furthermore, the amount of mature cross-links of collagen matrix was significantly reduced in GIPR deficient animals and was associated with lowered intrinsic material properties. Taken together, these data support a positive effect of the GIPR on bone strength and quality.
\end{abstract}

(c) 2013 Elsevier Inc. All rights reserved.

\section{Introduction}

Bone is a living mineralized material, highly complex and constantly remodeled in mass and architecture to adapt and repair the damage induced by growth, ageing and mechanical stress. In order to maintain constant bone mass, bone remodeling necessitates a spatio-temporal coupling between osteoclasts, the bone-resorbing cells, and osteoblasts, the bone-forming cells. Thus, bone remodeling is a complex process tightly regulated by a network of local, hormonal and neuronal factors that act on osteoclasts and osteoblasts. A role for the gastro-intestinal tract in bone remodeling has been suggested with the evidence that the modulation of serum markers of bone resorption is mirrored with the profile of gut hormone release after a meal $[1,2]$.

Glucose-dependent insulinotropic polypeptide (GIP) is an important gastro-intestinal hormone synthesized and secreted into the blood stream by intestinal endocrine $\mathrm{K}$ cells after ingestion of a mixed meal [3-5]. To induce a biological response, GIP binds to a

\footnotetext{
* Corresponding author at: GEROM-LHEA UPRES EA 4658, Institut de Biologie en Santé, Université d'Angers, 4 rue larrey, 49933 Angers Cedex 09, France. Fax: + 33244688451 E-mail address: guillaume.mabilleau@univ-angers.fr (G. Mabilleau).
}

specific glucose-dependent insulinotropic polypeptide receptors (GIPRs), expressed in the endocrine pancreas, gastrointestinal tract, brain, immune and cardiovascular systems, testis, pituitary, lung, kidney, thyroid, several regions of the central nervous system and adipose tissue [6]. The presence of a functional GIPR has been evidenced at the surface of osteoblasts and osteoclasts [7-10]. However, the role of GIP in bone physiology remains unclear. In vitro, it seems that GIP stimulates the synthesis of collagen type I and TGF- $\beta$ by osteoblasts $[11,12]$, whilst it directly inhibits RANKL-induced osteoclast bone resorption in raw 264.7 cells [10]. However, despite these in vitro results that suggest an anabolic effect of GIPR signaling in bone, inconsistent results have been reported regarding the physiological role of the GIP/GIPR pathway in vivo. Although Xie et al. reported a lower bone mass in a mouse model of GIPR deficiency [13], we recently reported, in a separate model of GIPR deletion, the opposite skeletal phenotype with an increase in trabecular bone volume associated with a modification of the adipokine network [14]. Nevertheless, in our model, we also observed that the intrinsic properties of the neo-synthesized trabecular bone matrix were decreased suggesting a possible effect of GIPR deficiency on bone strength [14].

Bone is a highly sophisticated connective tissue that is organized from the molecular to the anatomical level to resist and adapt to mechanical strain. As such several levels of organization account for bone 
strength and include not only material properties of the bone matrix, but also texture of the collagen matrix, bone microarchitecture and bone macroarchitecture [15]. However, little is known about how the GIP/GIPR pathway affects these different levels of organization in bone. The aims of the present study were to investigate bone strength and quality in male mice with genetic deletion of the GIPR. Our results suggest that GIPR-deficient mice have significant alterations of the cortical microarchitecture and material properties that undoubtedly result in reduced bone strength. These findings support a positive role for the GIP/GIPR signaling pathway in controlling bone strength and quality.

\section{Materials and methods}

\section{Animals}

Male mice presenting with a deletion of the GIPR were used in this study. The background and generation of GIPR-deficient mice used in this study were derived from an in-house breeding colony originally described elsewhere [16]. Sixteen weeks old mice were used in the study. Two animal models of GIPR deletion exist and age of the animals in the present study was chosen based on our previous observations of bone alterations in this model [14]. Age-matched wild-type (WT) mice obtained from our animal supplier (Harlan Ltd., Oxon, UK) with the same C57BL/6 genetic background were used as controls. Mice were maintained on a $12 \mathrm{~h}: 12 \mathrm{~h}$ light-dark cycle in a temperature-controlled room $\left(21.5 \pm 1{ }^{\circ} \mathrm{C}\right)$. Animals were individually caged and received food and water ad libitum. All experiments were conducted according to United Kingdom Office regulations (UK Animals Scientific Procedures Act 1986) and European Union laws. Animals were injected intraperitoneally with calcein $(10 \mathrm{mg} / \mathrm{kg})$ 7 and 2 days before necropsy. Animals were sacrificed by lethal inhalation of $\mathrm{CO}_{2}$ and left and right femurs were collected, cleaned of soft tissue and stored in $70 \%$ ethanol at $4{ }^{\circ} \mathrm{C}$.

\section{Quantitative x-ray microradiograph imaging}

Quantitative x-ray imaging has been shown to be a reliable method to assess the bone mineral content of whole bone and to correlate well with bone strength as assessed by 3-point bending [17]. Digital $\mathrm{x}$-ray images of the left femurs were recorded at a $12-\mu \mathrm{m}$ pixel resolution using a Faxitron MX20 device (Edimex, Angers, France) operating at $26 \mathrm{kV}$ and a $4 \times$ magnification. The relative mineral content of calcified tissues was determined as reported by Bassett et al. [18] with the following modifications. Briefly, a $1.5-\mathrm{mm}$ thick steel plate, a $1.5-\mathrm{mm}$ pure aluminum wire and a $1.5-\mathrm{mm}$ thick polyester plate were used on each microradiograph and served as standards. Before converting the 16-bit DICOM images into 8-bit tiff images, the histogram was stretched from the polyester (gray level 0 ) to the steel (gray level 255) standards using Image $1.45 \mathrm{~s}$. Increasing gradations of mineralization density were represented in 16 equal intervals using the 16-colors lookup table in Image $1.45 \mathrm{~s}$.

The frequency of occurrence of an $i$ gray level $\left(F_{i}\right)$ was calculated as follows:

$F_{i}=100 \times \frac{N_{i}}{N_{t}}$

where $N_{i}$ represents the number of pixels with the $i$ gray level and $N_{t}$ the total number of pixels. The distribution of frequency as a function of gray level was plotted and the mean gray level $\left(\mathrm{GL}_{\text {mean }}\right)$ of each bone was deduced from this distribution using the following formula:

$\mathrm{GL}_{\text {mean }}=\sum \frac{F_{i} \times \mathrm{GL}_{i}}{100}$

where $\mathrm{GL}_{i}$ represents the value of the $i$ gray level.

\section{$X$-ray microcomputed tomography}

MicroCT analysis was performed in the left femurs with a Skyscan 1172 microtomograph (Bruker-Skyscan, Kontich, Belgium) equipped with an X-ray tube working at $69 \mathrm{kV} / 100 \mu \mathrm{A}$. The pixel size was fixed at $3.75 \mu \mathrm{m}$, the rotation step at $0.25^{\circ}$ and exposure was performed with a $0.5-\mathrm{mm}$ aluminum filter. The region of interest (VOI) was located in the middle of the femur diaphysis. External bone diameter (B.Dm in $\mathrm{mm}$ ), marrow diameter (Ma.Dm in $\mathrm{mm}$ ), cortical thickness (Ct.Th in $\mu \mathrm{m}$ ), and cross-sectional moment of inertia (CSMI in $\mathrm{mm}^{4}$ ) were measured with a lab-based routine made with Image $1.45 \mathrm{~s}$ (NIH, Bethesda, MD) according to guidelines and nomenclature proposed by the American Society for Bone and Mineral Research [19].

\section{Bone histomorphometry}

Left femurs were embedded and undecalcified in poly (methylmethacrylate) (pMMA) at $4{ }^{\circ} \mathrm{C}$ to preserve enzyme activities. Sections (7- $\mu \mathrm{m}$ thickness) were cut on a heavy duty microtome equipped with a $50^{\circ}$ tungsten carbide knife. For each animal, four non serial sections $(\sim 50 \mu \mathrm{m}$ apart) were left unstained for the measurement of calcein-based parameters (original magnification $\times 400$ ) and four sections were stained for the osteoclastic tartrate resistant acid phosphatase (TRAcP - original magnification of $\times 200$ ) as previously described [20]. Only TRAcP-positive nucleated cells in contact with endosteal surface were counted as osteoclasts. Standard bone histomorphometrical nomenclatures, symbol and units were used as described in the report of the American Society for Bone and Mineral Research [21].

\section{Bone mechanical testing}

Three-point bending experiments were performed using right femurs. Before mechanical testing, femurs were rehydrated in saline for $24 \mathrm{~h}$ at room temperature as described elsewhere [22]. Threepoint bending strength was measured with a constant span length of $10 \mathrm{~mm}$. The press head as well as the two support points were rounded to avoid shear load and cutting. Femurs were positioned horizontally with the anterior surface facing upwards, centered on the support and the pressing force was applied vertically to the midshaft of the bone. Each bone was tested with a loading speed of $2 \mathrm{~mm} \mathrm{~min}{ }^{-1}$ until failure with a $90 \mathrm{~N}$ load cell. The load-time curve obtained was converted into a load-displacement curve by the MTS testSuite TW software (MTS, Créteil, France). Ultimate load and ultimate displacement were respectively defined as the maximum load and maximum displacement recorded before break-down of the bone. Stiffness was calculated as the slope of the elastic deformation of the bone. The total absorbed energy was defined as the total area under the load-displacement curve and represents the total energy absorbed by the midshaft femur. The yield was defined as the load necessary to initiate the transformation from elastic to plastic deformation. The post-yield energy was defined as the area under the load-displacement curve from yield until failure and represents the energy absorbed by bone during plastic deformation.

\section{Quantitative backscattered electron imaging ( $q B E I$ )}

Quantitative backscattered electron imaging was employed on the femurs to determine the bone mineral density distribution (BMDD) as previously reported $[23,24]$. Polymethylmethacrylate blocks were polished to a 1- $\mu \mathrm{m}$ finish with diamond particles, carbon-coated and observed with a scanning electron microscope (EVO LS10, Carl Zeiss Ltd., Nanterre, France) equipped with a five quadrants semi-conductor backscattered electron detector. The microscope was operated at $20 \mathrm{keV}$ with a probe current of $120 \mathrm{pA}$ and a working distance of 
$8.5 \mathrm{~mm}$. The backscattered signal was calibrated using pure carbon $(Z=6$, mean gray level $=25)$, pure aluminum $(Z=13$, mean gray level $=225)$ and pure silicium $(Z=14$, mean gray level $=253)$ standards (Micro-analysis Consultants Ltd., St. Ives, UK). For these contrast/ brightness settings, the BSE gray level histogram was converted into weight percentage of calcium. Eventual changes in brightness and contrast due to instrument instabilities were checked by monitoring the current probe and imaging the reference material ( $\mathrm{C}, \mathrm{Al}$ and $\mathrm{Si}$ ) every $15 \mathrm{~min}$. The cortical bone area was imaged at a $200 \times$ nominal magnification, corresponding to a pixel size of $0.5 \mu \mathrm{m}$ per pixel. The region of interest corresponded to 2-mm centered in the midshaft femur. The gray levels distribution of each image was analyzed with a lab-made routine using ImageJ. Three variables were obtained from the bone mineral density distribution: $\mathrm{Ca}_{\text {peak }}$ is the most frequently observed calcium concentration, $\mathrm{Ca}_{\text {mean }}$ is the average calcium concentration and $\mathrm{Ca}_{\text {width }}$ is the width of the histogram at half maximum of the peak.

\section{Nanomechanical testing}

Nanoindentation tests evaluated the intrinsic mechanical properties of the bone matrix. As nanoindentation assesses volume of material at a length scale less than that of individual microstructural features in bone, this technique avoids confounding factors such as bone microarchitecture and porosity that affect tissue properties at larger length scales. Tests were performed on the same sample and same location as qBEI measurements. Briefly, femurs were rehydrated overnight in saline prior to nanoindentation testing. Eight indents were positioned in cortical bone with a NHT-TTX system (CSM, Peseux, Switzerland) equipped with a Berkowitch diamond probe. The indents were made up to a depth of $900 \mathrm{~nm}$ with a loading/ unloading rate of $40 \mathrm{mN} / \mathrm{min}$. At maximum load, a holding period of 15 seconds was applied to avoid creeping of the bone material. Maximum load, indentation modulus, hardness and dissipated energy were determined according to Oliver and Pharr [25].

\section{FTIR}

Sections of $4 \mu \mathrm{m}$ thickness were cut dry on a heavy duty microtome equipped with a tungsten carbide knife (Leica Polycut $\mathrm{S}$ ) and sandwiched between $\mathrm{BaF}_{2}$ optical windows. Spectral analysis was performed using a Bruker Vertex 70 spectrometer (Bruker optics, Ettlingen, Germany) interfaced with a Bruker Hyperion 3000 infrared microscope equipped with a standard single element Mercury Cadmium Telluride (MCT) detector. Infrared spectra were recorded at a resolution of $4 \mathrm{~cm}^{-1}$, with an average of 32 scans in transmission mode in the same location as qBEI and nanoindentation. Background spectral images were collected under identical conditions from the same $\mathrm{BaF}_{2}$ windows at the beginning and end of each experiment to ensure instrument stability. For FTIRM analysis, 10 spectra were acquired 6-mm below the growth plate on cortical bone and analyzed with the Opus Software (release 5.5, Bruker). Sequential raw spectra for each trabecula were averaged and the contribution of the embedding polymethylmethacrylate (pMMA) and water vapor were corrected prior to baseline correction. The evaluated IR spectral parameters were (1) mineral-to-matrix ratio which reflects the degree of mineralization of the bone matrix, calculated from the ratio of integrated areas of the phosphate $v 1, v 3$ band at $900-1200 \mathrm{~cm}^{-1}$ to the amide I band at $1585-1725 \mathrm{~cm}^{-1}$; (2) mineral maturity, which reflects the apatite size and perfection, calculated as the ratio of the relative intensity of subbands at 1020 and $1030 \mathrm{~cm}^{-1}$ of the phosphate band [26]; and (3) collagen maturity, determined as the relative ratio of pyridinium trivalent (Pyr, mature collagen) to dehydrodihydroxylysinonorleucine divalent (deH-DHLNL, new collagen) collagen cross-links using their respective subbands located at $1660 \mathrm{~cm}^{-1}$ and $1690 \mathrm{~cm}^{-1}$ of the Amide I peak [27].

\section{Statistical analysis}

Results were expressed as mean \pm standard error of the mean (SEM). Non-parametric Mann-Whitney $U$-test was used to compare the differences between the groups using the Systat statistical software release 13.0 (Systat software Inc., San Jose, CA). Differences at $p<0.05$ were considered to be significant.

\section{Results}

\section{GIPR is required for optimum bone strength}

Bone strength of the femur was assessed by three-point bending and results are presented Table 1. In GIPR KO mice, ultimate load, stiffness, total absorbed and post-yield energies were reduced significantly by $11 \%, 16 \%, 28 \%$, and $27 \%$ respectively as compared with WT animals. No differences in either ultimate displacement or yield load were observed between the two groups of animals.

\section{GIPR-deficiency results in altered femoral mineral density and cortical geometry}

In order to further understand why bone strength was reduced in GIPR deficient animals, we investigated, by quantitative x-ray microradiographs, the bone mineral content of the whole femur and the cortical bone geometry. As represented Fig. 1A, bone mineral content seemed lower in GIPR KO animals and indeed, the frequency of occurrence of gray level, representing the bone mineral content, was shifted toward the left in GIPR KO mice indicating a lower bone mineral content (Fig. 1B). Furthermore, the mean gray level was significantly reduced by $5 \%$ in GIPR KO mice as compared with WT controls ( $p=0.037$, Fig. 1C). Microarchitectural analysis of cortical bone (Fig. 2) revealed that although B.Dm was not affected in GIPR KO animals as compared with WT controls, Ma.Dm was significantly increased by $10 \%$ in deficient animals $(p=0.043)$. Consequently, Ct.Th and CSMI were significantly lowered by $20 \%$ and $18 \%$ respectively in deficient animals as compared with WT $(p=0.021$ and $p=0.032$ respectively). The number of osteoclast per endosteal surface length (N.Oc/Ec.Le) was significantly increased by $213 \%$ in GIPR KO animals ( $p=0.034)$. On the other hand, the MAR was unchanged at endosteal surfaces between the two groups of animals $(p=0.564)$.

Intrinsic mechanical properties of the bone matrix are reduced in GIPR KO mice

Another important contributor of bone strength is represented by the intrinsic bone matrix properties. As compared with WT animals, GIPR-deficient animals exhibited a significant 13\% decrease in maximum load, as well as a significant $16 \%$ reduction in bone matrix hardness (Table 2). Although diminished, the indentation modulus and the energy dissipated were unchanged between the two groups of animals.

Table 1

Three-point bending parameters in WT and GIPR KO mice.

\begin{tabular}{|c|c|c|c|}
\hline & WT & GIPR KO & $p$ Value \\
\hline & $(n=12)$ & $(n=11)$ & \\
\hline Ultimate load $(\mathrm{N})$ & $14.47 \pm 0.35$ & $12.94 \pm 0.24^{*}$ & 0.034 \\
\hline Ultimate displacement ( $\mathrm{mm})$ & $0.31 \pm 0.006$ & $0.32 \pm 0.017$ & 0.827 \\
\hline Stiffness $\left(\mathrm{N} \mathrm{mm}^{-1}\right)$ & $42.68 \pm 0.85$ & $35.92 \pm 1.47^{*}$ & 0.05 \\
\hline Total absorbed energy ( $\mathrm{N} \mathrm{mm}$ ) & $2.33 \pm 0.09$ & $1.68 \pm 0.17^{*}$ & 0.05 \\
\hline Yield load $(\mathrm{N})$ & $11.83 \pm 0.45$ & $10.21 \pm 0.77$ & 0.127 \\
\hline Post-yield energy ( $\mathrm{N} \mathrm{mm}$ ) & $1.52 \pm 0.05$ & $1.11 \pm 0.27 *$ & 0.05 \\
\hline
\end{tabular}

Values are mean $\pm \operatorname{SEM}(n)^{*}: p<0.05$ vs. WT animals. 
A

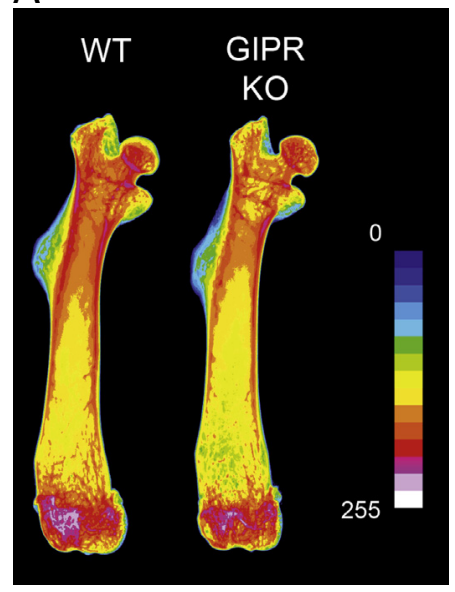

\section{$\mathrm{B}$}

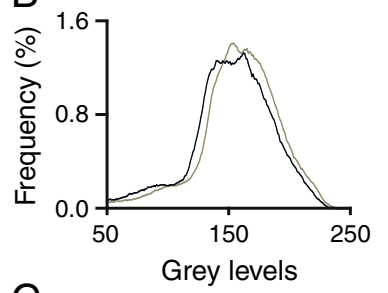

C

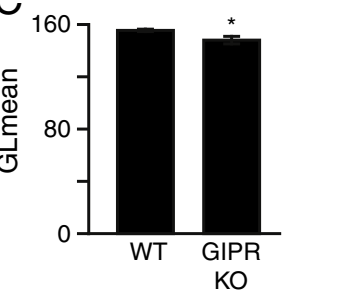

Fig. 1. Femoral bone mineral content is altered in GIPR KO animals. (A) Bone mineral content as determined by quantitative $\mathrm{x}$-ray microradiographs is reduced in GIPR KO animals as compared with WT. (B) Bone mineral content distribution is also reduced in GIPR KO mice (black line) as compared with WT control animals (gray line). (C) Mean gray level is also reduced in GIPR KO mice as compared with WT animals * $p<0.05$ vs. WT animals. $N=11-12$ in each group. (For interpretation of the references to color in this figure legend, the reader is referred to the web version of the article.)

\section{Collagen and mineral maturation are altered in GIPR KO mice}

In order to understand why the intrinsic mechanical properties of the bone matrix were reduced, we investigated the impact of GIPR deficiency on the maturation of the mineral and collagen compartments of the bone matrix. The bone mineral density distribution in the bone matrix was investigated by qBEI in WT and GIPR KO mice
(Table 3). Interestingly, GIPR-deficient animals seemed to present with a shift to the left of their distribution of mineralization (Fig. 3). Indeed as shown in Table $3, \mathrm{Ca}_{\text {peak }}$ and $\mathrm{Ca}_{\text {mean }}$ were significantly decreased by $12 \%$ and $11 \%$ respectively ( $p=0.018$ and $p=0.045$ respectively). In contrast, $\mathrm{Ca}_{\text {width }}$ was significantly elevated in deficient animals by $15 \%$ ( $p=0.044)$. The carbonate substitution of hydroxyapatite and the mineral maturity/cristallinity, although increased and decreased respectively as compared with WT control animals, did not reach significance $(p=0.195$ and $p=0.653$ respectively, Fig. 4). However, the ratio of trivalent mature over divalent immature collagen crosslinks was significantly decreased by $16 \%$ in GIPR KO mice as compared with WT $(p=0.003)$.

\section{Discussion}

The strength of bone, and its ability to resist fracture, is dependent not only on its mass and geometry but also on intrinsic material properties of the bone tissue itself. Bone tissue is a composite material consisting of an organic matrix, made mainly with collagen type I, in which mineral crystals are embedded. This composite material has mechanical, chemical and biological properties that differ considerably from those of either component taken separately. As such, any alteration in the organic or mineral phase of the bone matrix would result in altered bone strength. In the present study, we investigated bone strength in a mouse model of GIPR deficiency to understand how the GIP/GIPR pathway may affect bone strength. In the C57BL/6 mouse strain employed, bone tissue at the midshaft femur is composed almost exclusively of cortical bone $[28,29]$. Three-point bending experiments, performed on the midshaft femur, revealed a significant decrease in the total absorbed energy as well as reduction in ultimate load, stiffness and post-yield energy. These results suggest reduced bone strength in these animals. However, several factors
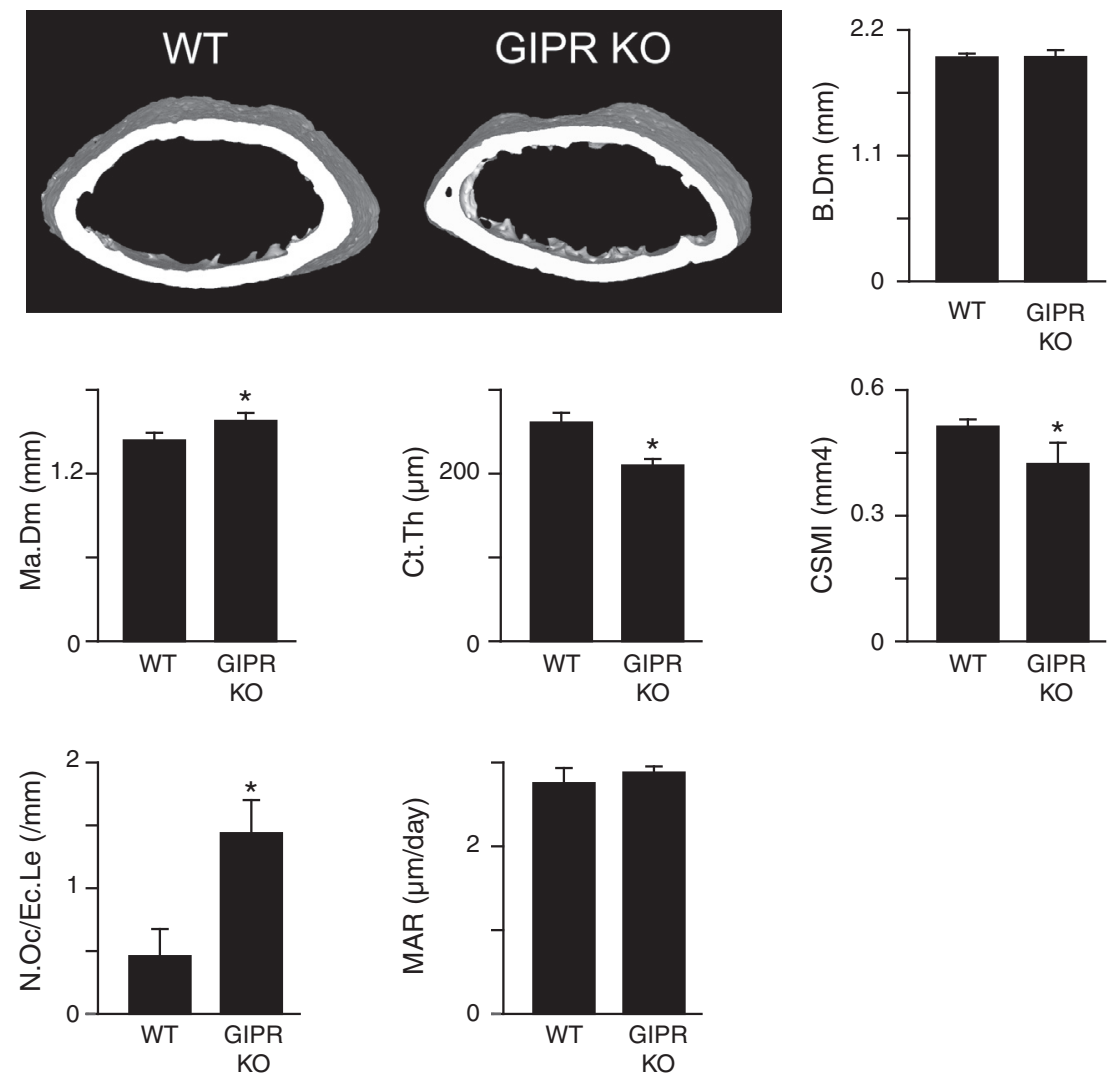

Fig. 2. 3-D models and histomorphometrical analysis of cortical bone in WT and GIPR KO mice. *: $p<0.05$ vs. WT animals. $N=11-12$ in each group. 
Table 2

Intrinsic properties of the bone matrix.

\begin{tabular}{lcccc}
\hline & WT & & GIPR KO & p Value \\
\cline { 2 - 2 } \cline { 5 - 5 } & $(n=12)$ & & $(n=11)$ & \\
\hline Maximum load (mN) & $11.9 \pm 0.6$ & & $10.4 \pm 0.5^{*}$ & 0.032 \\
Hardness (MPa) & $627.8 \pm 34.4$ & & $537.6 \pm 37.2^{*}$ & 0.043 \\
Indentation modulus (GPa) & $12.9 \pm 0.6$ & & $12.1 \pm 0.4$ & 0.253 \\
Dissipated energy (mN nm) & $3189.7 \pm 176.7$ & & $2917.0 \pm 123.7$ & 0.153 \\
\hline
\end{tabular}

Values are mean $\pm \operatorname{SEM}(n) *: p<0.05$ vs. WT animals.

might influence the outcome of three-point bending including bone microarchitecture and bone matrix intrinsic properties [30].

Inconsistent results have been previously reported on the trabecular microarchitecture of GIPR deficient mice, but very little is known about the effect of the GIP/GIPR on cortical bone microarchitecture. In the present study, we investigated cortical bone microarchitecture by high resolution $\mu \mathrm{CT}$ and observed a significant increase in Ma.Dm, whereas B.Dm was unchanged. As a consequence, CT.Th and CSMI were also decreased compatible with the lower bone strength observed by three-point bending. As bone formation occurs at periosteal surfaces and bone resorption at endosteal surfaces, these results suggest that bone resorption is more affected than bone formation in GIPR deficient animals. Furthermore, to add to our understanding of the role of osteoclastic bone resorption in these animals, we observed that the number of osteoclasts lying down on endosteal surfaces was significantly increased in deficient animals, also suggesting higher bone resorption. Previous reports suggest that the GIPR is expressed in osteoblasts and osteoclasts [7-10], and GIP appeared to dose-dependently reduced osteoclast resorption in RANKL- and PTH-stimulated Raw 264.7 cells [10]. Therefore the observation that Ma.Dm is increased in GIPR knockout animals is compatible with a direct effect of the GIP/GIPR pathway on osteoclasts. Nevertheless, indirect effects on bone resorption mediated by neuronal or hormonal actions due to GIPR deletion in other tissues than bone cannot be ruled out. Indeed, the GIPR is expressed in the hypothalamus and this organ has been implicated in the central control of bone remodeling by directly targeting bone cells. As such, the increased Ma.Dm could also result from activation/inactivation of specific central relays. Furthermore, we previously reported that GIPR deficient animals present with a significant reduction in fat mass in association with remodeling of circulating adipokines with specific elevation of adiponectin and reduction of leptin levels [14]. The effects of leptin on bone are complex and result from integration of hypothalamic and peripheral signals. Nevertheless, mice with functional mutation in the gene for leptin present with a low cortical bone mass in the femur [31,32]; this is associated with an augmented number of osteoclasts at the endosteal surface. As such, the decrease in circulating leptin levels observed in our GIPR KO model [14] might result in lower cortical bone mass as observed.

Another factor that strongly affects bone strength is the intrinsic quality of the bone matrix [22]. GIPR KO mice presented with a decrease in maximum load $(-17 \%)$, hardness $(-19 \%)$ and dissipated energy $(-15 \%)$. Owing to the nanocomposite composition of bone matrix, any of the collagen or mineral phases could be involved in the reduction in intrinsic material properties. Investigation of bone mineral properties by qBEI and FTIR revealed that the degree of mineralization of the bone matrix was lower in GIPR deficient animals.

Table 3

Bone mineral density distribution in WT and GIPR deficient mice.

\begin{tabular}{lccl}
\hline & WT $(n=12)$ & GIPR KO $(n=11)$ & $p$ Value \\
\hline $\mathrm{Ca}_{\text {peak }}(\%)$ & $26.9 \pm 0.7$ & $23.7 \pm 0.9^{*}$ & 0.018 \\
$\mathrm{Ca}_{\text {mean }}(\%)$ & $26.3 \pm 0.6$ & $23.4 \pm 0.9^{*}$ & 0.045 \\
$\mathrm{Ca}_{\text {width }}(\%)$ & $2.7 \pm 0.1$ & $3.1 \pm 0.1^{*}$ & 0.044 \\
\hline
\end{tabular}

Values are mean $\pm \operatorname{SEM}(n)^{*}: p<0.05$ vs. WT animals.

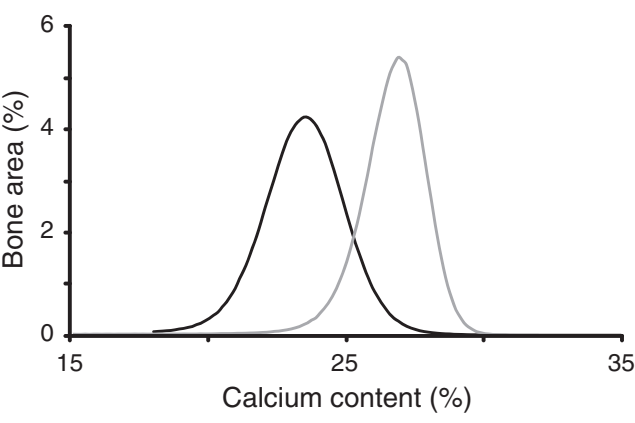

Fig. 3. Bone mineral density distribution in GIPR KO (black line) and WT (gray line) animals. $N=11-12$ in each group.

This observation could represent either a lower content of mature mineral in the bone matrix or a less mature hydroxyapatite that results in lower calcium content. The carbonate substitution of hydroxyapatite, although increased, did not reach statistical significance and similar effects were noted with decreased mineral maturity. Taken together, these results support a less mature mineral matrix. This is also in agreement with the increased bone remodeling observed at endosteal surfaces. Nevertheless, we did not assess the biochemical composition of the bone matrix (mainly proportion of type I collagen and relative composition in non-collagenous proteins) in control and GIPR KO mice. Thus we cannot exclude a difference in the biochemical composition that could influence the mineralization rate of the bone matrix. Furthermore, the maturity of the collagen matrix, determined as a ratio of trivalent mature Pyr over divalent immature deH-DHLNL, was significantly reduced indicating a lower degree of maturation of the collagen matrix. The reduction of bone mineral content and trivalent mature crosslinks help account for the reduction in intrinsic material properties. Furthermore, alteration of the collagen matrix often alters the post-yield properties of the bone matrix [33-35], in agreement with the present study where the reduction in mature trivalent crosslinks could account for the observed decrease in post-yield energy determined by 3-point bending.

We previously demonstrated that intrinsic material properties of the bone matrix in cancellous bone of GIPR-deficient animals were altered [14]. It is nevertheless worth noting that some differences in intrinsic material properties between cancellous and cortical bone exist in this animal model. First of all, the reduction in collagen crosslink ratio was greater in cancellous bone $(\sim 42 \%)$ as compared with cortical bone $(\sim 16 \%)$. Furthermore, although heterogeneity of bone mineral density distribution $\left(\mathrm{Ca}_{\text {width }}\right)$ was not significantly reduced in cancellous bone, this parameter was considerably increased in cortical bone. Also, the decrease in $\mathrm{Ca}_{\text {peak }}$ and $\mathrm{Ca}_{\text {mean }}$ was greater in cortical bone. In other words, it seems that alteration of collagen maturity was greater in cancellous bone whilst alteration of mineral maturity was greater in cortical bone. Finally, in cancellous bone, the indentation modulus
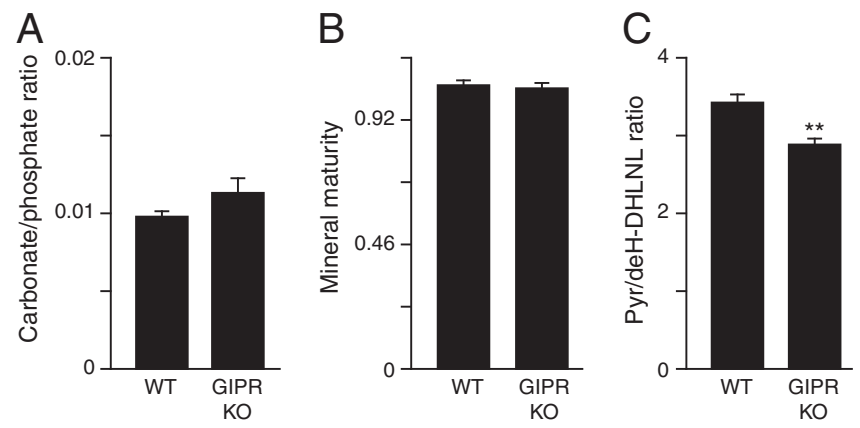

Fig. 4. FTIR assessment of bone matrix properties. (A) Carbonate substitution, (B) mineral maturity and $(C)$ enzymatic collagen cross-link ratio. ${ }^{* *}: p<0.01$ vs. WT animals. $N=11-12$ in each group. 
and dissipated energy were significantly reduced whilst unaltered significantly in cortical bone. It is plausible that discrepancies in these two last parameters between cancellous and cortical bones might reflect the differential modifications of the two bone matrix components although further studies are required to undoubtedly validate that hypothesis. A limitation of this study resides in the choice of controls animals. Control animals are issued from a commercial source (Harlan) and are not littermates. as such we cannot exclude that the observed phenotype has arisen from mutations in either strain that might contribute to the observed reduction in bone strength.

In conclusion, mice lacking the GIPR exhibited a decrease in anatomical bone strength with a similar decrease in three-point bending resistance and cortical thickness. This was accompanied by an increase in bone resorption. Bone strength was also reduced at the tissue level and was associated with reductions in the degree of mineralization, maturity of the hydroxyapatite mineral and collagen crosslinking. Overall these data support a beneficial positive role for the GIP/GIPR signaling pathway in strength and quality of cortical bone.

\section{References}

[1] Elnenaei MO, Musto R, Alaghband-Zadeh J, Moniz C, Le Roux CW. Postprandial bone turnover is independent of calories above $250 \mathrm{kcal}$. Ann Clin Biochem 2010;47:318-20.

[2] Henriksen DB, Alexandersen P, Bjarnason NH, Vilsboll T, Hartmann B, Henriksen $\mathrm{EE}$, et al. Role of gastrointestinal hormones in postprandial reduction of bone resorption. J Bone Miner Res 2003;18:2180-9.

[3] Andersen DK, Elahi D, Brown JC, Tobin JD, Andres R. Oral glucose augmentation of insulin secretion. Interactions of gastric inhibitory polypeptide with ambient glucose and insulin levels. J Clin Invest 1978;62:152-61.

[4] Schirra J, Katschinski M, Weidmann C, Schafer T, Wank U, Arnold R, et al. Gastric emptying and release of incretin hormones after glucose ingestion in humans. J Clin Invest 1996;97:92-103.

[5] Varner AA, Isenberg JI, Elashoff JD, Lamers CB, Maxwell V, Shulkes AA. Effect of intravenous lipid on gastric acid secretion stimulated by intravenous amino acids. Gastroenterology 1980;79:873-6.

[6] Baggio LL, Drucker DJ. Biology of incretins: GLP-1 and GIP. Gastroenterology 2007; 132:2131-57.

[7] Bollag RJ, Zhong Q, Phillips P, Min L, Zhong L, Cameron R, et al. Osteoblast-derived cells express functional glucose-dependent insulinotropic peptide receptors. Endocrinology 2000;141:1228-35.

[8] Mabilleau G, Gaudin-Audrain C, Irwin N, Flatt PR, Basle MF, Chappard D. Deficiency in glucose-dependent insulinotropic peptide receptor results in higher bone mass in male mice. Osteoporos Int 2012;23:S407-8.

[9] Pacheco-Pantoja EL, Ranganath LR, Gallagher JA, Wilson PJ, Fraser WD. Receptors and effects of gut hormones in three osteoblastic cell lines. BMC Physiol 2011;11:12.

[10] Zhong Q Itokawa T, Sridhar S, Ding KH, Xie D, Kang B, et al. Effects of glucose-dependent insulinotropic peptide on osteoclast function. Am J Physiol Endocrinol Metab 2007;292:E543-8.

[11] Bollag RJ, Zhong Q, Ding KH, Phillips P, Zhong L, Qin F, et al. Glucose-dependent insulinotropic peptide is an integrative hormone with osteotropic effects. Mol Cell Endocrinol 2001;177:35-41.

[12] Zhong Q Ding KH, Mulloy AL, Bollag RJ, Isales CM. Glucose-dependent insulinotropic peptide stimulates proliferation and TGF-beta release from MG-63 cells. Peptides 2003;24:611-6.
[13] Xie D, Cheng H, Hamrick M, Zhong Q Ding KH, Correa D, et al. Glucose-dependent insulinotropic polypeptide receptor knockout mice have altered bone turnover. Bone 2005;37:759-69.

[14] Gaudin-Audrain C, Irwin N, Mansur S, Thorens B, Flatt PR, Basle MF, et al Glucose-dependent insulinotropic polypeptide receptor deficiency leads to modifications of trabecular bone mass and quality in mice. Bone 2013;53:221-30.

[15] Chappard D, Basle MF, Legrand E, Audran M. New laboratory tools in the assessment of bone quality. Osteoporos Int 2011;22:2225-40.

[16] Preitner F, Ibberson M, Franklin I, Binnert C, Pende M, Gjinovci A, et al Gluco-incretins control insulin secretion at multiple levels as revealed in mice lacking GLP-1 and GIP receptors. J Clin Invest 2004;113:635-45.

[17] Bassett JH, Gogakos A, White JK, Evans H, Jacques RM, van der Spek AH, et al Rapid-throughput skeletal phenotyping of 100 knockout mice identifies 9 new genes that determine bone strength. PLoS Genet 2012;8:e1002858.

[18] Bassett JH, van der Spek A, Gogakos A, Williams GR. Quantitative X-ray imaging of rodent bone by Faxitron. Methods Mol Biol 2012;816:499-506.

[19] Bouxsein ML, Boyd SK, Christiansen BA, Guldberg RE, Jepsen KJ, Muller R. Guidelines for assessment of bone microstructure in rodents using micro-computed tomography. J Bone Miner Res 2010;25:1468-86.

[20] Chappard D, Alexandre C, Riffat G. Histochemical identification of osteoclasts. Review of current methods and reappraisal of a simple procedure for routine diagnosis on undecalcified human iliac bone biopsies. Basic Appl Histochem 1983;27:75-85.

[21] Parfitt AM, Drezner MK, Glorieux FH, Kanis JA, Malluche H, Meunier PJ, et al. Bone histomorphometry: standardization of nomenclature, symbols, and units. Report of the ASBMR Histomorphometry Nomenclature Committee, 2. J Bone Miner Res; 1987. p. 595-610.

[22] Ammann P, Badoud I, Barraud S, Dayer R, Rizzoli R. Strontium ranelate treatment improves trabecular and cortical intrinsic bone tissue quality, a determinant of bone strength. J Bone Miner Res 2007;22:1419-25.

[23] Gaudin-Audrain C, Irwin N, Mansur S, Flatt PR, Thorens B, Baslé M, et al Glucose-dependent insulinotropic polypeptide receptor deficiency leads to modifications of trabecualr bone mass and quality in mice. Bone 2013;53:221-30.

[24] Roschger P, Fratzl P, Eschberger J, Klaushofer K. Validation of quantitative backscattered electron imaging for the measurement of mineral density distribution in human bone biopsies. Bone 1998;23:319-26.

[25] Oliver WC, Pharr GM. An improved technique for determining hardness and elastic modulus using load and displacement sensing indentation experiments. J Mater Res 1992;7:1564-83.

[26] Paschalis EP, DiCarlo E, Betts F, Sherman P, Mendelsohn R, Boskey AL. FTIR microspectroscopic analysis of human osteonal bone. Calcif Tissue Int 1996;59:480-7.

[27] Paschalis EP, Verdelis K, Doty SB, Boskey AL, Mendelsohn R, Yamauchi M. Spectroscopic characterization of collagen cross-links in bone. J Bone Miner Res 2001:16:1821-8.

[28] Bouxsein ML, Myers KS, Shultz KL, Donahue LR, Rosen CJ, Beamer WG. Ovariectomyinduced bone loss varies among inbred strains of mice. J Bone Miner Res 2005;20: 1085-92.

[29] Judex S, Garman R, Squire M, Donahue LR, Rubin C. Genetically based influences on the site-specific regulation of trabecular and cortical bone morphology. J Bone Miner Res 2004;19:600-6.

[30] Turner CH, Burr DB. Basic biomechanical measurements of bone: a tutorial. Bone 1993;14:595-608.

[31] Burguera B, Hofbauer LC, Thomas T, Gori F, Evans GL, Khosla S, et al. Leptin reduces ovariectomy-induced bone loss in rats. Endocrinology 2001;142:3546-53.

[32] Hamrick MW, Pennington C, Newton D, Xie D, Isales C. Leptin deficiency produces contrasting phenotypes in bones of the limb and spine. Bone 2004;34:376-83.

[33] Bailey AJ, Sims TJ, Ebbesen EN, Mansell JP, Thomsen JS, Mosekilde L. Age-related changes in the biochemical properties of human cancellous bone collagen: relationship to bone strength. Calcif Tissue Int 1999;65:203-10.

[34] Burr DB. The contribution of the organic matrix to bone's material properties. Bone 2002;31:8-11.

[35] Zioupos P, Currey JD, Hamer AJ. The role of collagen in the declining mechanical properties of aging human cortical bone. J Biomed Mater Res 1999;45:108-16. 